\title{
Research of Time Irreversibility in Voltage Mode Controlled DCM Buck Converter
}

\author{
Hongmei Xu \\ Institute of Engineering \\ Yanbian University \\ Yanji, China \\ e-mail: hmxu@ybu.edu.cn \\ Jiaqi Liu \\ Institute of Engineering \\ Yanbian University \\ Yanji, China \\ e-mail: 1193279872@qq.com
}

\author{
Aobo Wang \\ Department of electronic information engineering \\ Harbin Institute of Technology \\ Harbin, China \\ e-mail:wangaoboqwe915@sina.com
}

\begin{abstract}
This paper takes a voltage feedback DCM Buck converter as example, and analysis the distance in the converter which apart from equilibrium based on symbolic dynamics and relative entropy. The result shows that the relative entropy can quantify the distance of converter in chaos state, which can provide new ideas and methods for investigating the complexityof the nonlinear dynamics in converters.
\end{abstract}

Keywords-buck converter; nonlinear dynamics; symbolic dynamics;relative entropy

\section{INTRODUCTION}

Chaos theory originates from the meteorological phenomena by Lorenz[1]. Then the Chua's circuit opens the new chapter of the search of piecewise smooth circuit chaos phenomenon, which is differential multi-volume wave circuit that when the circuit parameter changes, the piecewise circuit structure and configuration complexity are changeless. DC/DC converter is a piecewise smooth circuit which use the diode or nonlinear devices such as filed effect tube by PWM way. when circuit structure changes, the circuit structure complexity will change at the same time, and it cannot product multi-volume wave chaotic state, so we must study on chaos state according to the characteristics of the switching converter itself. Many scholars have adapt qualitative researches on the chaotic characteristics of switching converter by using state space average[2], Jacobian[3], and parameter perturbation[4]. But qualitative viewpoint to analyze switching converter chaotic system is not enough to fully understand the essential characteristics of chaos state. We still need to search for quantitative describe chaos state of switching converter in order to precisely analyze the influence of different parameters of switching converter chaos system and complexity of different state. so the further understand the internal regularity and forecast control of the switching converter can be obtained.

Characteristic of the chaos system have spacious space by using entropy theory. Reference[5] discuss the unimodal map topological entropy of chaos state, and the universality of the topological entropy for one-dimension map is discussed. Reference[6] demonstrate the relationship between numerical distribution information entropy of switching converter in period state and chaos state. Reference[7] have made the analysis of the nonlinear characteristic of electroencephalogram, and it conclude that multiscal permutation entropy could discrimanate the electroencephalogram between normal and epileptic.

Symbolic analysis has been widely used, such as medicine, electrochemistry and secrete communication. Symbolic dynamic theory has provide the feasibility for the quantitative analysis of the chaos characteristics of switching converter. Reference[8] judge the symbol array composed by duty ratio of the critical state, and chaos state derives from border collision. Lempel-Ziv complexity based on the symbols array have quantitative describe the abrupt point and cycle converter[9].

The origanism combination of the information entropy and symbol dynamic could quantify the complexity of chaotic sequence. Reference [10] propose block entropy of symbol series by numericl series, and demonstrate that block entropy could accurately distinct period bifurcation and chaos behavior of DC/DC converter; Reference [11] calculate spectral entropy of three typical chaotic systems with different initial values and system parameters, and show spectral entropy could effectively analyze structure complexity of chaos series. However, block entropy could do nothing to quantize the distance which conveter deviate from its equilibrium point in chaos state; power spectrum entropy can separate period and chaos state, but the power spectrum which based on Fourier transform may reduce the frequency resolution ratio and cause the sidelobe leak, and cannot quantize the system non-equilibrium state. Porporate put forward the relative entropy to quantized the state that the system deviated from equilibrium[12], and seek the rationality of the used of relative entropy in converter. This paper give the example of voltage control of discontinuous mode (DCM) Buck converter, which is based on symbol dynamic and relative entropy, to quantized the distance which switch converter deviate from equilibrium state, and provide the new thought and ways to improve the nonlinear characteristics of switch converter. 


\section{RELATIVE ENTROPY THEORY}

Relative entropy is the distance between two random distribution. Relative entropy can be obtained based on probability distribution and Shannon entropy, the algorithm is discribed as follows:

Step1: For the symbol sequence of the length $L$, the forward sequence and back sequence is coded based on sliding module method, code length is $m$.

Step2: Calculate the probability distribution $P_{f}(s)$ and $P_{b}(s)$ of the forward sequence and back sequence. The sum of $P_{f}(s)$ and $P_{b}(s)$ is respectively 1 . For any $s$, it must satisfy the condition of $P_{f}(s) \geq 0$ and $P_{b}(s) \geq 0$.

Step 3: Calculate the relative entropy:

$$
D\left(P_{f} \| P_{b}\right)=\sum_{s \in S} P_{f}(s) \log \frac{P_{f}(s)}{P_{b}(s)}
$$

Where, $S=2^{m}, m$ is code length. If $P_{f}(s)=0$ or $P_{b}(s)=0$, eqn(4) is 0 . As a result, relative entropy is only the function for calculating or distance, and not symmetrical, that is $D\left(P_{f} \| P_{b}\right) \neq D\left(P_{b} \| P_{f}\right)$.

\section{RELATIVE ENTROPY OF DCM BUCK CONVERTER}

Nowadays, the widely used model of switching converter is put forward by Tse[15]. The voltage controlled Buck converter is discussed in this paper. When the converter work in discontinuous inductor current state, we can treat it as first-order system. According to the symbol sequence which is generated by the different feedback gains $k$ and initial value $x_{0}$ to calculate the relative entropy, so we can achieve the result which quantify the distance that switching converter deviate from the equilibrium point in chaos state.

\section{A. Numerical symbol sequence}

DCM Buck converter is shown in Fig .1. $E$ is input voltage, $X$ is output voltage, $D$ is duty ratio, $k$ is feedback proportion gain, and converter's working condition can be approximately described as first-order iteration map:

$$
x_{n+1}=f\left(k, x_{n}\right)=\alpha x_{n}+\frac{\beta\left[D-k\left(x_{n}-X\right)\right]^{2} E\left(E-x_{n}\right)}{x_{n}}
$$

where, $x_{n}$ is capacity voltage at the moment which inductance current is 0 , other parameters are illustrated as follows:

$$
\begin{gathered}
\alpha=1-\frac{T}{R C}+\frac{T^{2}}{2 C^{2} R^{2}} \\
\beta=\frac{T^{2}}{2 L C}
\end{gathered}
$$

$$
h\left(d_{n}\right)=\left\{\begin{array}{l}
0, \quad d\left(x_{n}\right)<0 \\
1, \quad d\left(x_{n}\right)>0 \\
d\left(x_{n}\right), \quad \text { other }
\end{array}\right.
$$

$$
d\left(x_{n}\right)=D-k\left(x_{n}-X\right)
$$

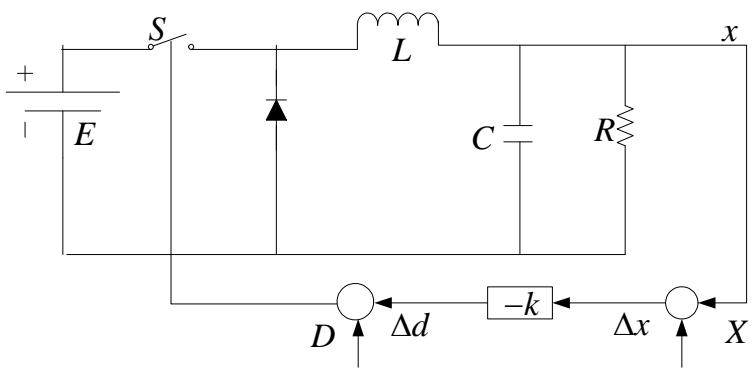

Figure 1. Diagram of DCM Buck converter

The converter adopt the parameters in the document [15], $T=333.33 \mu \mathrm{s}, E=16 \mathrm{~V}, C=222 \mu \mathrm{F}, R=12.5 \Omega$, $L=208 \mu \mathrm{H}, X=25 \mathrm{~V}, D=0.2874, \alpha=0.8872$, $\beta=1.2$, DCM Buck converter regard the feedback gain $k$ as variable quantity ,and the bifurcation is shown in Fig .2. When the converter is in chaos state, we can obtain the inverse single peak mapping which is shown in Fig .3. The lowest point $C=26.4$ of inverse single peak mapping function is the critical point, and the rise branch is " 0 ", and the descend is " 1 ", so the disperse numerical sequence $\left\{s_{0}, s_{1}, s_{2}, \ldots, s_{n} \ldots\right\}$ is obtained, and the complex dynamics characteristic analysis can be changed into relatively easy symbol sequence to study.

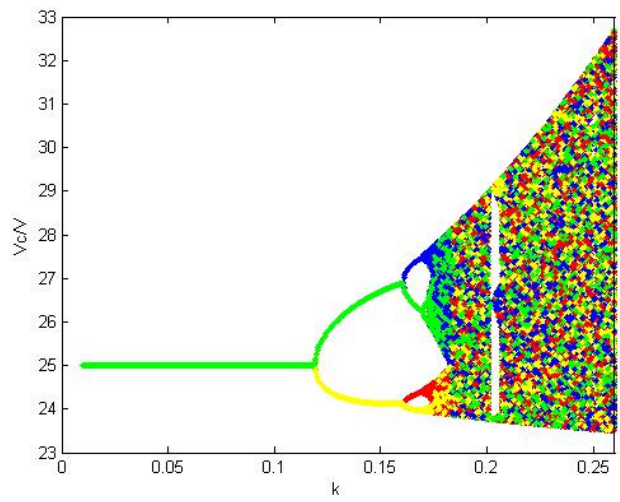

Figure 2. Bifurcation of DCM Buck converter 


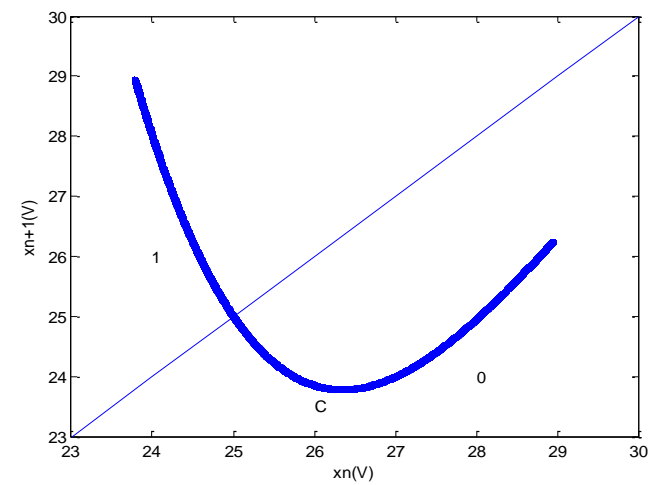

Figure 3. Return map of DCM Buck converter $(\mathrm{k}=0.22)$

\section{B. Calculate relative entropy}

The simulation experiment of calculating DCM Buck converter relative entropy is proceeded in the condition of Matlab 7.0. The initial value $x_{0}$ is in [23.27,31.99], the sequence length is 3000 according to the reference[16], code length is 8 bit, the range of relative entropy value is shown in Fig .4. Combine with Fig .2, the information reflected in Fig .4 is discribed as follows:

i )when $k=0.1032$, converter is in stable state, and the relative entropy is 0 .

ii ) When $k=0.1512$, converter is in period state, it will appear the numerical value composed by 0 and 1 after symbolization and the disperse relative values after coding. Relative entropy only achieve the $10^{-3}$ orders due to the few states.

iii) When $k=0.1991$, converter is in chaos state, so relative entropy is continue, and change in the range of $[0$, 0.08 ].

iv ) When $k=0.2012$, relative entropy appear to decline and go back to the $10^{-3}$ orders, and some zero state emerge.
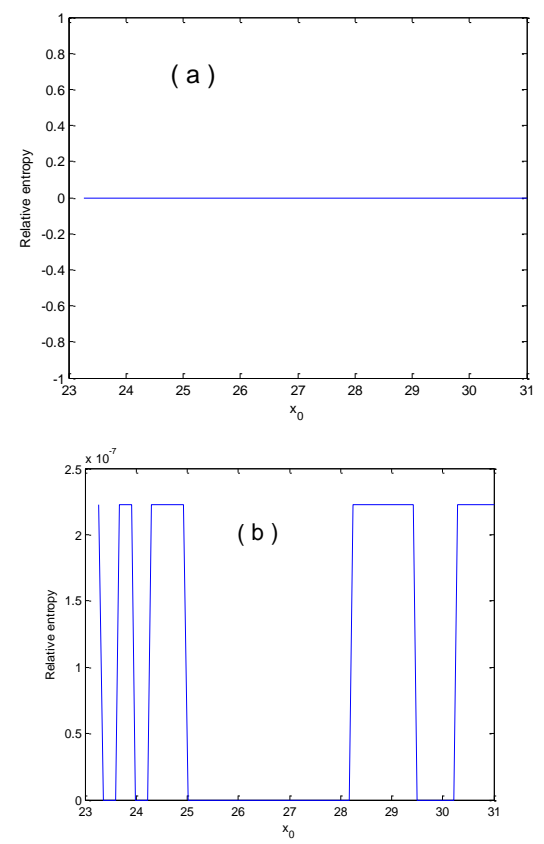
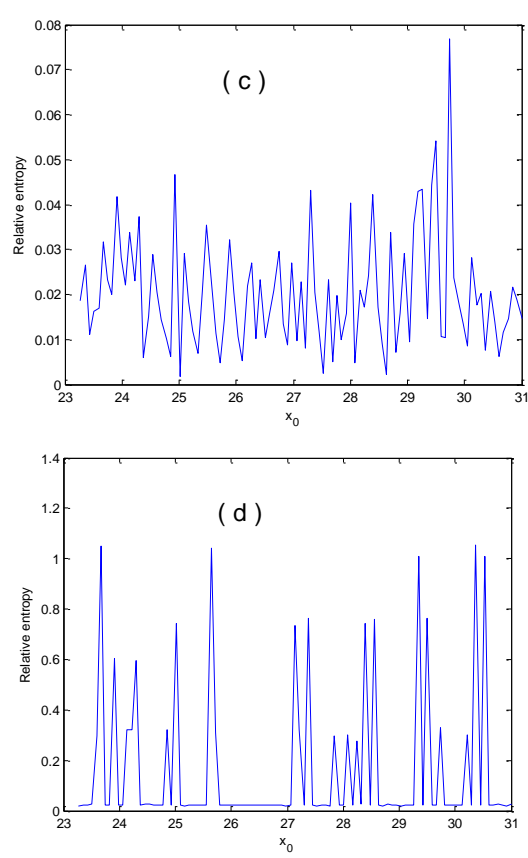

Figure 4. Relative entropy of different parameter k(a) k=0.1032 (b) $\mathrm{k}=0.1512$ (c) $\mathrm{k}=0.1991$ (d) $\mathrm{k}=0.2012$

For further to research the relative entropy range as parameter change, gain parameter $k$ is in the range of $[0.100,0.256]$ and initial value $x_{0}$ is in the range of [23.27,31.99] according to Fig .2. The chaos sequence can be obtained by (1), all of the numbers will not to be deleted and take part in calculate, and the three-dimensional drawing can be obtained in Fig .5. The result can be obtained based on Fig .5:

i) different $k$ corresponds to different working state;

ii) when converter is in unstable state, relative entropy is 0 . In the period state, the relative entropy maintain in $10^{-3}$ orders of magnitude. Converter will turn into chaos state through multi-period bifurcation state as the value of feedback ratio gain parameter $k$ increasing, the variation range of relative entropy will amplify. When $k$ is in [0.2010,0.2037], bifurcation will turn into period 3, it appear tangent lead to the numbers of switching converter concentrate in the range of some numerical points, and explain the reason of the value of relative entropy notable decline in the increasing progress. When $k$ greater than 0.204 , the converter turn into completely chaos state according to period 3 meaning chaos theory, and the corresponding entropy value relatively large. Beyond the abrupt point, the range of chaos sequence become bigger with the increase of parameter $k$, the degree of converter chaos system deviate from equilibrium state become deeper, so the sequence of relative entropy become bigger with the increase of parameter $k$.

iii) There are some abrupt point in the entropy increasing process, that is at the specific feedback ratio gain parameter $k$, switch converter iteration mapping is not in chaos state, but stop at period bifurcation state, and the numbers are dense, all of the numbers are concentrate 
in some statistics range lead the abrupt points corresponding entropy value increase rapidly.

iv) When parameter $k$ greater than 0.25 , relative entropy will become decline trend on the contrary. When parameter $k$ reach certain value, converter is in ergodic chaos state, and convert numerical sequence into symbols series, the differences between forward and back sequence become smaller, so relative entropy decrease.

v) In the value range of feedback ratio gain parameter $k$, the trend of DCM Buck converter relative entropy value change is the same as the different initial value $x_{0}$, so it can prove that relative entropy does not depends on initial value when converter in chaos state.

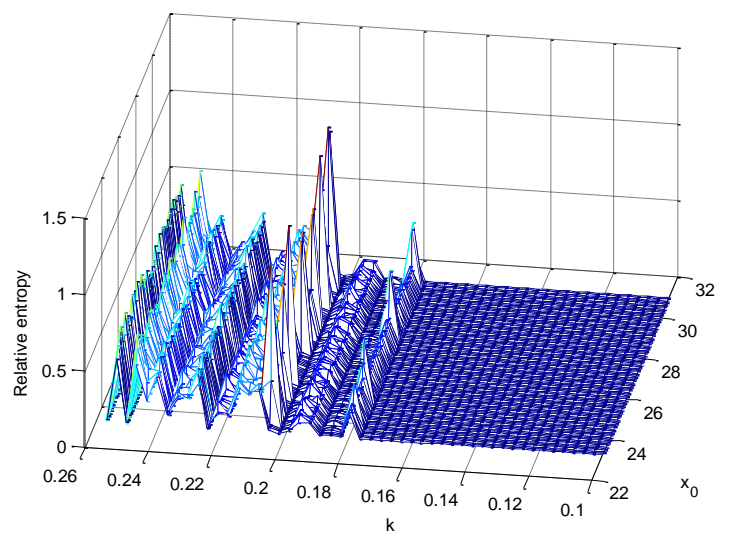

Figure 5. 3D relative entropy of DCM Buck converter

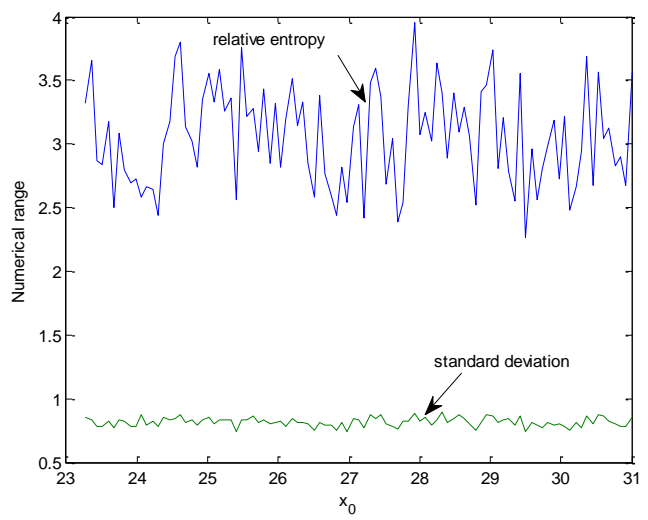

Figure 6. Relative entropy variable and standard deviation with different $\mathrm{x} 0$

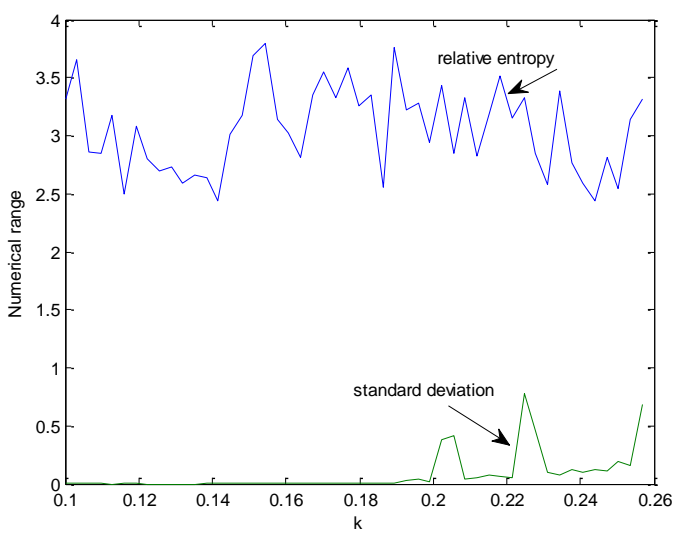

Figure 7. Relative entropy variable range and standard deviation of different $\mathrm{k}$

\section{CONCLUSIONS}

This passage take analysis and simulation of voltage controlled DCM Buck converter relative entropy theory with different parameters, and prove that use relative entropy theory to analysis switch converter deviate from equilibrium point at chaos state have obvious physical meanings, it quantify the level that the system deviate from equilibrium state when feedback parameter $k$ change on the based of remain the original system dynamic characteristics at the maximum limit, which can quickly and efficiently extract the useful quantitative information from the chaos series. Relative entropy could accuratly analysis the distance that converter deviate from equilibrium point in chaos state, symbol sequence length and embedded dimension have excellent robustness, it can fluctuate within a certain scope and maintain relative stability, for the more complex chaos state, the relative entropy fluctuate range is relatively small, thus we could make reasonable predictions for the trend of DC/DC converter chaos state, and provide new idea and method to reveal the nonlinear characteristics of switching converter.

\section{ACKNOWLEDGMENT}

The research work are supported by Natural Science Foundation of Yanbian University under Grant No. 2012800-602014044 and Scientific Research Starting Foundation of Yanbian University under Grant No. 2012800-602014093.

\section{REFERENCES}

[1] D.Y. Chen, C. Wu, H. C. Herbert, X.Y. Ma. Circuit simulation for synchronization of a fractional-order and interger-order chaotic system. Nonlinear Dynamics, vol.73, pp.1671-1686, Mar, 2013.

[2] A. Marconato, J. Sjiberg, J.A.K. Suykens, J. Schoukens. Improved initialization for nonlinear state-space modeling. IEEE Transactions on instrumentation and Measurement, vol.63, pp.972980, Apr. 2014.

[3] D.Giaouris, S. Banerjee, O. Imrayed, et al..Complex interaction between tori and onset of three-frequency quasi-periodicity in a current mode controlled boost converter. IEEE Transactions on Circuit and Systems-I, vol.59, pp.207-214, Jan.2012.

[4] G.L. Li, C.Y. Li, X.Y. Chen, X.W. Zhang. Chaos control of SEPIC converter based on resonant parametric perturbation method. Acta Physica Sinica, vol.62,pp.210505, (21)2013. 
[5] R.X. Chen and S.G. Chen. The topological entropy of onedeimensiona unimodal maps. Acta Physica Sinica, vol.35, pp.13381346, Oct.1986.

[6] H.M. Xu, Y.G. Jin, and S.X. Guo. Entropy in voltage mode controlled discontinuous conducting mode DC-DC converters. Acta Physica Sinica, vol.62, pp. 248401, Dec.2013.

[7] W.P. Yao, T.B. Liu, J.F. Dai, and J. Wang. Multiscale permutation entropy analysis of electroencephalogram. Acta Physica Sinica, vol.63, pp.078704, Jul.2014.

[8] D. Dai, X.K. Ma, and X.F. Li. Border collision bifurcations and chaos in a class of piecewise smooth systems with two boundaries Acta Physica Sinica, vol.52, pp. 2729-2736, Nov.2003.

[9] F. Xie, R.Yang, B. Zhang. Analysis of weight Lempel-Ziv complexity in piecewise smooth systems of DC-DC switching converters. Acta Physica Sinica, vol.61, pp.110504, Nov.2012.

[10] X.M. Wang, B. Zhang, and D.Y. Qiu. The Quantitative characterization of symbolic series of a boost converter. IEEE Transactions on Power Electronics, vol.26, pp.2101-2105, Aug. 2011.
[11] K.H. Sun, S.B. He, Y. He, et cl.. Complexity analysis of chaotic pseudo-random sequences based on spectral entropy algorithm. Acta Physica Sinica, vol.62, pp. 010501,Jan.2013.

[12] A. Porporato, J. R. Rigby, and E. Daly. Irreversibility and fluctuation theorem in stationary time series. Physical Review Letters, vol.98, pp.094101, Sep.2007.

[13] H.B. Jiang, T. Li, X.L. Zeng and L.P. Zhang. Bifurcation analysis of the logistic map via two periodic impulsive forces. Chinese Physica B, vol.23, pp. 010501. Jan.2014.

[14] Z. Rached, F. Alajaji, and L. L. Campbell. The Kullback-Leibler divergence rate between markov sources. IEEE Transactions on Information Theory, vol.50,pp.917-921, May.2004.

[15] C. K.Tse. Flip bifurcation and chaos in three-state boost switching regulators. IEEE Transactions on Circuit and Systems-I, vol.40,pp.16-23, Jan.1994.

[16] H.M. Xu, and S.X. Guo. Time irreversibility analysis of Logistic chaos system based on symbolic relative entropy. Journal of Electromics \& Information Theory,vol.36,PP.1241-1246, May. 2014 . 